\title{
Norois
}

Environnement, aménagement, société

$222 \mid 2012$

Xynthia

\section{Zones construites, zones désertes sur le littoral atlantique. Les leçons du passé}

Built zones, desert zones on the Atlantic littoral. Lessons of the past

\section{Martine Acerra et Thierry Sauzeau}

\section{CpenEdition}

1 Journals

Édition électronique

URL : https://journals.openedition.org/norois/4048

DOI : 10.4000/norois. 4048

ISBN : 978-2-7535-1843-8

ISSN : $1760-8546$

\section{Éditeur}

Presses universitaires de Rennes

\section{Édition imprimée}

Date de publication : 28 février 2012

ISBN : 978-2-7535-1815-5

ISSN : 0029-182X

\section{Référence électronique}

Martine Acerra et Thierry Sauzeau, «Zones construites, zones désertes sur le littoral atlantique. Les leçons du passé », Norois [En ligne], 222 | 2012, mis en ligne le 30 mars 2014, consulté le 13 janvier 2022. URL : http://journals.openedition.org/norois/4048; DOI : https://doi.org/10.4000/norois. 4048

(c) Tous droits réservés 


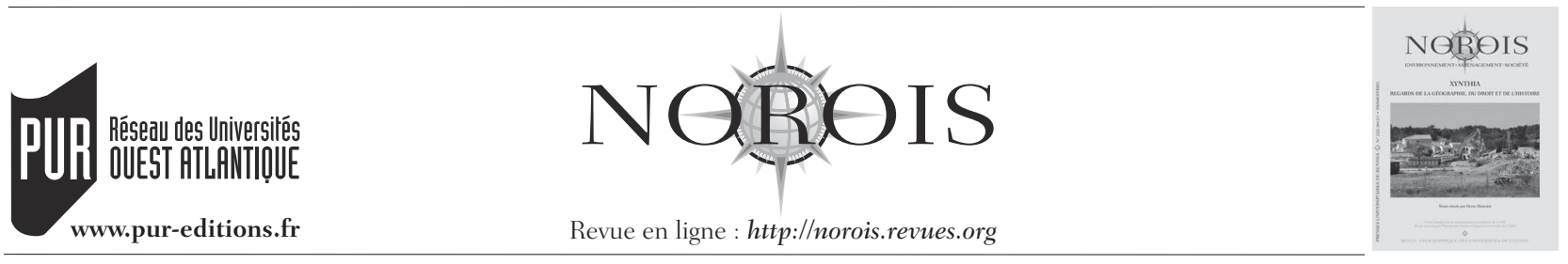

\title{
Zones construites, zones désertes sur le littoral atlantique Les leçons du passé
}

\author{
Built zones, desert zones on the Atlantic littoral \\ Lessons of the past
}

\author{
Martine ACERra $^{\mathrm{a} *}$, Thierry SAUZEau ${ }^{\mathrm{b}}$
}

\begin{abstract}
* auteur correspondant : tel : $33(0) 240141105$. fax : $33(0) 240141423$
a CRHIA - EA 1 163, Centre de Recherches en Histoire International et Atlantique, Université de Nantes, Chemin

de la Censive-du-Tertre, BP 81227, 44312 NANTEs Cedex 3, France (martine.acerra@univ-nantes.fr)

b CRIHAM - EA 4270, Centre de Recherches Interdisciplinaires Histoire, Histoire de l'Art et Musicologie,

Université de Poitiers, 36 rue de La Chaine, 86022 Poitıens Cedex, France (thierry.sanzeau@univ-poitiers.fr)
\end{abstract}

Résumé : Cet article explore la question des relations entre les installations en zone littorale, la mémoire locale et les connaissances officielles sur l'environnement littoral. Sous l'angle historique, cette étude peut débuter sous le règne de Louis XIV. Les archives officielles témoignent de l'intérêt de Colbert pour la mémoire locale dans le processus de décision de l'installation du port et arsenal de Rochefort. La Révolution Française a modifié ce mode de décision et la Monarchie Libérale (1830-1848) en a fondé un autre. Ainsi, les autorités ont laissé les communautés littorales libres d'aménager des marais et des polders par elles mêmes, en intervenant le moins possible. Le siècle suivant (1850-1950) a connu un accroissement du contrôle administratif. La mémoire locale a été collectée et connectée aux progrès scientifiques afin de donner naissance à une doctrine officielle de développement littoral. La croissance du tourisme a définitivement brisé ce modèle, en substituant une société urbaine et sans racines aux modes de vie traditionnels et anciens, réglés par la mémoire locale. Au même moment, les technologies numériques ont établi un monopole sur la formation des ingénieurs. Cela a contribué à faire avancer la connaissance des littoraux mais cela a aussi accéléré la perte de la mémoire locale, particulièrement celle des risques côtiers.

\begin{abstract}
This article explores the question of relationships between coastal settlements, inhabitants memory and official knowledges about littoral environment. From an historical point of view, the study begins during the reign of Louis 14. The official archives show that Colbert was interested in collecting people's experience so as to decide where and how would be created the new harbour and arsenal of Rochefort by the monarchy. Such decision process has been changed by the French Revolution and a new one was built during the Liberal Monarchy (1830-1848). Therefore, the authorities let the littoral communities free to make up polders and marshes, with few interventions. The following century (1850-1950) has been a time for an increasing adminitrative control. The strong local experience has been collected and connected to the science's progress so as to create an official doctrine of coastal development. The take-off of the tourism has definitly broken this model, replacing the old and traditionnal way of life - ruled by the local memory - by an urban and rootless society. At the same time, the monopoly of numeric technologies on ingeneer's sciences has become a reality. Althought it seems to be a good way to fill some gap of knowledges, it has also accelerated the loss of experience and local memory, specialy about maritime risks.
\end{abstract}

Mots clé : littoral - savoir - mémoire - histoire - aménagements - Xynthia

Keywords : coastline - knowledge - memory - history - installations - Xynthia 


\section{INTRODUCTION}

Cet article a pour objectif de souligner, à partir d'une documentation officielle couvrant les périodes moderne et contemporaine, la prudence de répartition de la population littorale en des temps où le risque à vivre sur les côtes faisait partie des mémoires et la récente construction d'une vulnérabilité littorale. À partir des archives, de l'iconographie, des cartes, plans, correspondances et autres discours manuscrits, il est possible de percevoir un enchaînement de causes et d'effets concernant l'occupation de la frange côtière. Depuis la Renaissance, le roi et son pouvoir centralisateur tentent de «soumettre le rivage » (Zysberg, 1995). Pour ce faire, ils doivent apprendre à connaître le territoire frontalier de la mer bordant le royaume de France sur deux de ses faces atlantique et méditerranéenne. Quels que soient les motifs poussant à ces investigations, développées alors que la plupart des provinces maritimes sont tardivement rattachées au royaume, ils ont pour conséquence une remarquable recension du littoral comprenant ses diverses configurations, ses occupations et ses aménagements humains ${ }^{1}$. L'apogée de cet apprentissage du rivage se situe sans doute vers 1830, lorsque Charles François BeautempsBeaupré livre une cartographie très précise des côtes occidentales de la France (Taillemite, 2002). Aménagements portuaires, urbains ou balnéaires, cette œuvre fondatrice de l'hydrographie moderne va permettre l'expression du désir de rivage (Corbin, 1988). C'est le temps où la carte vient préciser le corpus de connaissances empiriques patiemment consigné par les hommes du roi sous l'Ancien Régime. Les dossiers instruits par l'administration des Ponts et Chaussées tout au long de la période allant de 1830 à 1980 portent la marque de cette double filiation. Le témoignage et la carte resteront longtemps les instruments d'aide à la décision privilégiés, avant d'être rejoints par la modélisation numérique, à l'extrême fin du $\mathrm{xx}^{\mathrm{e}}$ siècle.

Nous avons saisi ces opportunités documentaires pour développer trois idées : quelle qu'en soit la raison, un État soucieux d'efficacité doit savoir et connaître pour décider et agir; cet apprentissage passe par des acquis de terrain collectés auprès des pratiquants de l'espace concerné, afin que les élites

1. La Guyenne est rattachée en 1453, le comté de Provence en 1481, le duché de Bretagne à partir de 1532. décisionnelles acquièrent une solide connaissance des usages locaux; l'éventuelle emprise de l'État sur ce territoire par ce biais informatif n'évite pas les réticences et les conflits, l'orientation des réponses des usagers peu dupes des intentions venues d'en haut.

\section{RECUEILLIR L'EXPÉRIENCE DES RIVERAINS POUR PENSER LA MER}

Le début du règne de Louis XIV offre un excellent observatoire à notre propos. Sortant de multiples crises internes et externes, le royaume de France et son jeune souverain fort ambitieux peuvent « penser la mer ", et en conséquence, décliner une politique maritime totale. Celle-ci passe inévitablement par le recensement des multiples richesses littorales. Les années 1660-1670 sont celles de la formation de la France au monde de la mer, même si elles sont précédées par des périodes de géniales intuitions et de tentatives réitérées, le tout englobant un quart de siècle décisif. "L'époque couvrant les années 1630-1690 peut être considérée comme l'étape essentielle, au cours de laquelle l'État royal s'appropria le domaine maritime, transformant une souveraineté nominale en une tutelle effective qui portait sur l'espace, sur les hommes, sur les navires et sur les ports » (Zysberg, 1995).

\section{La monarchie absolue et l'appropriation officielle du savoir local}

Nos remarques concernent le littoral atlantique. Il présente une différence sensible par rapport au rivage méditerranéen pour ce qui regarde l'occupation humaine. Depuis la plus haute antiquité, les populations vivant près des côtes méditerranéennes ont appris à se méfier, non pas tant des colères de la mer, que des invasions répétées des pirates, razzieurs et autres prédateurs, qu'ils soient barbaresques ou non. De ce fait, les hommes se concentrent prudemment et plus volontiers dans les villes et villages bien protégés, s'installent sur les hauteurs permettant une vigilance visuelle. Ils se placent donc le plus souvent en retrait du rivage. En atlantique, non sujette à la répétition de ces agressions sans en être cependant à l'abri, l'installation humaine s'étale plus volontiers à proximité immédiate du littoral, en 
contact avec la ressource marine, jamais très loin de la grève, de l'estran, de l'estuaire, des richesses du sel et de la pêche.

Cependant, l'habitat côtier tient compte de la configuration géographique des lieux et prospère à l'abri des risques naturels les plus connus dont les montées des eaux font partie. L'exemple de la Charente suffira à illustrer cette prudence ancestrale (figure 1 - planche III). Le long du cours aval du fleuve jusqu'à l'estuaire, les villages et hameaux sont installés sur les enrochements, sur les «terres hautes » qui sont les anciennes îles du golfe de Saintonge, surplombant de quelques mètres le niveau de la mer. Les terres basses formées des marais inhospitaliers, insalubres et inondables à chaque marée, sont évitées. Le phénomène se vérifie dans le temps, malgré le début de la politique d'assèchement des marais menée sous l'impulsion royale d'Henri IV et ses successeurs, politique reprise des moines du XIII ${ }^{\mathrm{e}}$ siècle qui l'avait initiée. Aux alentours de la Charente, comme en d'autres lieux sur le littoral atlantique - Seudre, presqu'île d'Arvert, îles de Ré et d'Oléron - les zones côtières contenant des marais ou d'anciens marais, voient leurs habitats regroupés en villages ponctuant le modeste sommet des terres hautes ${ }^{2}$.

En réalité, les marais désertés ne sont qu'apparemment inhabités. Malgré l'hostilité du milieu, les plus fragiles économiquement y demeurent les pieds dans l'eau, dans des huttes de roseaux, tirant du marais de quoi survivre. Par ailleurs, au quotidien comme en périodes d'exceptionnelles répressions, les marais et les marécages offrent des lieux de refuge. Ils abritent toute une population occasionnelle faite de fuyards, de déserteurs, de protestants, de réfractaires. Non sans paradoxe, ces espaces mal aisés et dangereux du point de vue sanitaire, protègent ceux qui s'y cachent de risques encore plus grands.

D'abord perçu par portions provinciales, le littoral devient progressivement un ensemble à consi-

2. Ce n'est que dans les années récentes de notre ère que la pression immobilière a commencé à faire descendre les maisons neuves dans les anciens marais, ajoutant aux risques inhérents à ce choix la dégradation patrimoniale d'un paysage jusqu'alors authentique. Sans la vigilante volonté des Élus et de leurs administrés, le mouvement serait irréversible. La dérive est parfois parée lorsque, comme dans le cas de Hiers-Brouage, sont convoqués les lois et les instances qui, à la demande d'une équipe municipale courageuse, inscrivent dans la ZPPAUP l'ensemble du marais ainsi inconstructible et protégé. (Approbation du conseil municipal du 26 juillet 2007 ; inscription effective en 2008, CRPS de Poitou-Charentes). dérer dans sa globalité pour les souverains français et Louis XIV en particulier, même si la complexité et la variété du rivage ne leur échappent pas. La Guyenne, la Provence, la Bretagne ayant complété le littoral français depuis le $\mathrm{XV}^{\mathrm{e}}$ siècle, elles suscitent l'application d'une politique d'État faite, entre autres de créations portuaires (figure 2 - planche III). Puis, cette appropriation ponctuelle évolue vers le goût d'une soumission continue de la frontière maritime du royaume.

Pour connaître son littoral, le roi s'en remet aux initiatives de ses grands conseillers maritimes, Richelieu pour Louis XIII, Colbert pour Louis XIV. L'un invente ce que l'autre développera avec frénésie : l'enquête croisée, dans laquelle le recours aux savoirs des anciens sièges en bonne place comme source d'informations et bases efficaces de décision. Les enquêtes de 1632 et 1636 sur les côtes du Ponant et du Levant constituent le brouillon que Colbert va s'efforcer de perfectionner avec celles qu'il diligente en 1664 et $1665^{3}$.

En parallèle à cette vaste enquête de 1665 confiée aux sièges des amirautés, une autre inspection est effectuée sur ordre royal. Elle est le fait de " commissaires départis par Sa Majesté " nommés en novembre 1664. Il s'agit en fait des hommes de confiance de Colbert dont son propre cousin Charles Colbert de Terron, des deux ingénieurs des fortifications Chastillon et Clerville, de l'architecte François Blondel et d'un spécialiste hollandais de l'hydraulique, Régnier Jansse. Leur mission d'inspection «de tous les ports et havres de l'océan bordant la France » commence aux côtes de Dunkerque et s'achève à La Rochelle. Ils en rédigent un procèsverbal de visite le 15 avril 1665 qui sert à la mise en forme de la réponse officielle datée de Brouage, le premier mai suivant ${ }^{4}$.

Même si la concomitance de ces deux opérations visant des buts très proches illustre ce procédé des enquêtes croisées, il ne nous importe pas ici de décrire par le menu la technique utilisée par le futur secrétaire d'État à la marine de Louis XIV. Il convient cependant d'en retenir ce qui fait les délices de l'historien d'aujourd'hui, sa " religion du détail ». Ainsi, la précision du questionnaire de

\footnotetext{
3. Service Historique de la Défense, Vincennes, manuscrit SH 48, recueil des procès-verbaux de visites des ports et havres de France en application de l'arrêt du roi du 24 janvier 1665, 508 fo recto verso (non publié).

4. Archives du Génie, article 4, section 2, paragraphe 1, carton 1. Procèsverbal de la visite, fait à Brouage le 15 avril 1665 (non publié).
} 
1665 accompagnant l'ordre royal révèle-t-elle déjà les intentions. Mais les réponses des amirautés permettent de dresser un tableau du littoral français vu par les contemporains, usagers de ses espaces. Tableau parfois succinct, parfois complet, souvent inattendu.

L'arrêt du Conseil du Roi du 24 janvier 1665 ordonne la visite et la sonde des havres et ports de France. Les résultats de ces visites doivent faire l'objet « d'un procès-verbal du bon ou mawvais état auquel ils se trouveront». Les officiers de l'amirauté et autres officiers exerçant les causes maritimes chacun dans l'étendue de son ressort sont requis pour cette tâche. Ils doivent, entre autres, «entendre les plus anciens des lieux et experts qu'ils pourront nommer d'office pour avoir leur avis sur l'ancien état des ports et les différences apparues avec l'état d'aujourd'hui ». Il est donc fait appel à la mémoire et aux témoignages des aînés dans le but d'améliorer l'état des ports et en conséquence l'activité commerciale maritime du royaume.

À cette requête royale, Colbert ajoute la précision d'un questionnaire en trente huit points qui va bien au-delà de l'objectif initial et unique. Ecrites de sa propre main, les questions peuvent être regroupées en deux catégories et plusieurs thématiques. La première catégorie développe, en seize questions, la demande royale en exigeant la description de la structure naturelle du port, les aménagements existants, les améliorations possibles, en y ajoutant la production d'un plan. La seconde catégorie répond aux préoccupations colbertiennes en matière de protection militaire du port, de ressources matérielles et humaines (onze questions), de fiscalité locale.

D'un domaine particulier, le questionnaire fait passer l'enquête de 1665 à un audit du littoral, d'où transpirent quelques sous-entendus : trouver les auteurs des éventuelles dégradations; percevoir les querelles d'usage; souligner les droits usurpés ; protéger les ressources nationales; prendre en mains le littoral, zone stratégique de la plus haute importance.

Les réponses des quarante-huit amirautés constituent un dossier de 508 folios recto verso, dont les écritures et les longueurs varient d'un site à l'autre 5 .

5. Cette difficulté explique que le dossier n'a fait l'objet que d'exploitations partielles. Une exploitation globale de ce remarquable corpus est envisagée. Actuellement un étudiant en histoire du master recherche de l'université de Nantes, Mathieu Flora, participe à la mise en œuvre d'une
Les réponses parfois précises et détaillées permettent de souligner la finesse des observations effectuées. La qualité des hommes sollicités n'est pas moins intéressante.

Aux vastes questions sur la configuration portuaire répondent les remarques sur les enrochements naturels, les digues et les môles artificiels, l'impétuosité des flots, la protection contre les vents, les marées, les corsaires. Aux nombreuses interrogations concernant la présence des gens de mer, des bons matelots, charpentiers, pilotes, géographes et hydrographes, correspond une litanie de tristesse et d'abandon des activités halieutiques ou commerciales. D'autant plus que ces remarques négatives sont mises en miroir avec les temps jadis beaucoup plus heureux et prospères.

Chaque réponse de chaque secteur est éclairante, comme dans le cas des Sables-d'Olonne (figure 3 planche III). Les autorités locales ont interrogé les plus anciens usagers du lieu soit dix-sept « anciens bourgeois et maîtres de navires » qui décrivent l'univers qu'ils connaissent depuis trente à trente-cinq ans. Ils évoquent le port lui-même, décrivent le jeu contraignant des eaux douces en hiver, des fortes marées, des vents qui agitent les sables, des courants qui modifient les fonds. Ils soulignent aussi la pauvreté et la misère des habitants, la diminution du cabotage, la désertification de quelques petits ports désormais «inhabités ». Leur témoignage est authentifié par leurs années de pratique du lieu. Il en est de même pour les onze «anciens et principaux manants et habitants du bourg de Saint Gilles et de celui de Croix de Vie », également interrogés sur leur domaine d'activité maritime ${ }^{6}$.

Sur d'autres portions du littoral atlantique d'entre Loire et Gironde, le même souci de s'adresser à ceux qui savent est attesté. Il en est ainsi lorsque les officiers du siège d'amirauté des côtes et îles de Saintonge réunissent à Brouage cinq maîtres et pilotes de navire du lieu. Le plus âgé Antoine Riveau, 80 ans, dit qu'il y a "soixante ans et plus qu'il navigue dans le havre dudit Brouage ». Jean Boucher, son compère de 62 ans annonce quarante ans de pratique locale tandis que les trois plus jeunes, âgés de 36 à 47 ans, revendiquent aussi une lon-

analyse collective de ce bel ensemble, pour laquelle tous les chercheurs intéressés, historiens ou non, sont les bienvenus.

6. Service Historique de la Défense, Vincennes, manuscrit SH 48, Procèsverbal de visite des Sables-d'Olonne commencé le 15 avril 1665, fo 27 et suivants (non publié). 
gue connaissance de ces eaux. Leurs dires et témoignages sont croisés avec ceux d'un capitaine des vaisseaux du roi que l'on fait venir expressément de l'île d'Oléron ${ }^{7}$. Les sondes de la rade sont effectuées les jours suivants grâce à l'aide de quatre autres maitres pilotes de navire.

À La Rochelle, ce sont douze pilotes de la ville et neuf maîtres de barque qui livrent leur expertise et connaissance des rades et « rivières de Charente, Brouage, Seudre, courault d'Oléron et de La Pallice, l'Aiguillon et rades de Saint-Martin de Ré ${ }^{8} »$. Leur notoriété semble certaine et leur savoir reconnu puisqu'ils ont été «environ vingt ans auparavant » requis pour les mêmes types d'expertises. Le procès-verbal de l'amirauté de La Rochelle demeure cependant assez succinct, s'en tenant aux sondes des diverses rades visitées, sans répondre d'une quelconque façon au questionnaire de Colbert. Nous y apprenons ce que confirment d'autres textes ou plans (figure 4 - planche IV) pour l'anse d'Aiguillon : «À l'embouchure de la rivière de Marans il y a environ quatre brasses d'eau de pleine mer et quand le mer est basse, il n'y en a point du tout. »

L'enquête de 1665 marque un moment important dans la chaîne d'accumulation des savoirs concernant le littoral. Menée en parallèle d'autres investigations et d'autres origines de savoirs y compris étrangers ${ }^{9}$, elle peut être évaluée à l'aune de quelques-unes de ses conséquences. Le repérage du secteur primordial en matière de ressources humaines que constitue le littoral entre Loire et Gironde en est une. L'expérimentation du régime des classes et la première tentative de recensement des gens de mer sont menées sur ces côtes de l'Aunis - Saintonge. La richesse humaine locale et l'abondance de la production agricole tiennent leur rôle dans le choix de la Charente et de la terre de Rochefort pour y fonder un grand établissement de marine digne de Louis XIV (Acerra, 1993).

Rappelons aussi que cet audit, comparé aux enquêtes menées vingt ans plus tard sous l'égide de Seignelay, digne fils de Colbert et héritier de sa charge de secrétaire d'État à la marine, permet de

7. Service Historique de la Défense, Vincennes, manuscrit SH 48, PV des côtes et îles de Saintonge, fo 155 et suivants (non publié).

8. Service Historique de la Défense, Vincennes, manuscrit SH 48, PV de La Rochelle, fo 177 et suivantes (non publié).

9. La présence du Hollandais Régnier Jansse, signalée à de nombreuses reprises dans la correspondance ministérielle de Colbert, n'est pas innocente. souligner le redressement des activités maritimes de la France. Il n'est de richesse que d'hommes écrivait Richelieu en son temps. Remarque qui dépasse largement l'aspect quantitatif de la démographie et concerne aussi l'aspect qualitatif de la transmission des savoirs acquis.

\section{Les Lumières ou la décision éclairée par l'expérience du terrain}

En matière de politique littorale, le XVIII ${ }^{\mathrm{e}}$ siècle a largement hérité des pratiques inaugurées sous Louis XIV. Sous la monarchie administrative, plusieurs mémoires, rapports ou enquêtes d'envergure nationale attestent de cette même volonté d'écouter les riverains. L'œuvre du géographe du Roi Claude Masse (1696-1721) en fournit un premier exemple, même si elle s'apparente davantage aux oisivetés qu'affectionnait Vauban qu'à une commande officielle. En effet, durant ses chevauchées pour prendre les repères destinés à cartographier les frontières du royaume, Claude Masse a aussi rédigé des Mémoires géographiques ${ }^{10}$ dont le caractère pluridisciplinaire fait la part belle à l'économie, à l'ethnologie et aux descriptions des littoraux, des ports et des paysages qui les encadrent. Évoquant La Tremblade, port-chenal exposé au risque d'envasement, Masse indique ainsi que l'on «l'entretient par les eaux des marais qui vienne du côté des Mattes ».

Dans le secteur des pêches, l'enquête de l'inspecteur Le Masson du Parc, a quant à elle été conduite dans les années 1720 avec l'appui local des commissaires aux classes de la Marine et en multipliant les interrogatoires de pêcheurs, village après village ${ }^{11}$. Dans ce rapport, les préoccupations environnementales, tournées en priorité vers les ressources halieutiques, n'étaient pas exclusives de réflexions sur les aménagements côtiers, face aux forçages naturels (érosion, accumulation de sable ou de vases). Visitant le nord d'Oléron où les écluses à poissons étaient bâties en dur sur l'estran - en totale contravention avec les textes - Le Masson souhaitait que «le nombre en fût augmenté de manière [à] servir de digue et rompre les lames qui rongent le terrain » des falaises exposées aux houles d'ouest.

10. Médiathèque de La Rochelle, fonds ancien, Manuscrit géographique de Claude Masse (1720), Ms.31, copie réalisée par le père Jaillot (16901749), (non publié).

11. Archives départementales de Charente-Maritime, Enquête de l'inspecteur des pêches Le Masson du Parc (1727), 4 J 3440, (non publié). 
Enfin, à la veille de la Révolution française, Louis XVI confiait au commissaire de la Marine Chardon le soin de conduire une enquête sur les ports avec des préoccupations mi-économiques, mi-fiscales ${ }^{12}$. Ses consultations n'excluaient pas les questions environnementales, notamment au sujet du délestage des navires marchands, coupable d'accélérer l'envasement des ports saintongeais. On apprend ainsi qu'à la fin du XVIII ${ }^{\mathrm{e}}$ siècle, on avait fait «sur la Seudre aux frais des propriétaires des salines un canal qui facilite l'entrée des navires dans les terres afin d'y jeter le lest avec plus de précautions; mais quand ces bâtiments sont trop grands pour mouiller dans ce canal, les maîtres sont obligés de se servir de chaloupes qui reçoivent à flot leur lest et qui les portent au plus haut de l'eau sur les bords élevés de la rivière ou du courant d'Oléron".

Les interlocuteurs privilégiés étaient les officiers des amirautés. Institution typique de l'Ancien Régime, ces tribunaux maritimes disséminés en une cinquantaine de sièges sur tout le littoral étaient peuplés d'un personnel recruté chez les élites locales. Propriétaires de leur charge de judicature, les officiers d'Amirauté étaient le plus souvent intéressés à l'économie locale par le biais de leurs affaires ou encore par l'exercice de charges complémentaires (notariat, collecte des impôts, etc.). Par ailleurs, cette institution que la Révolution devait abolir en 1791, gérait au quotidien le dossier environnemental en exerçant la surveillance des pêches, en organisant le délestage des flottes marchandes, en contrôlant des prélèvements de matériaux ou l'exploitation du couvert végétal des dunes, des plages ou de l'estran.

Avant la Révolution française, le pilotage par l'État des questions environnementales sur le littoral s'effectuait donc grâce à la Marine, à travers ses bureaux des classes dans chaque quartier, mais plus encore grâce à l'Amirauté, par l'intermédiaire de ses sièges particuliers. Ces deux institutions disposaient de relais dans la société littorale en la personne des syndics de gens de mer ou de pêcheurs et d'un réseau de garde-côtes (Schnakenbourg, 1975; Péret, 2006) comme autant de courroies de transmission des règles à respecter mais aussi d'intermédiaires précieux pour la consultation des riverains sur les affaires qui les concernaient. À aucun moment,

12. Archives départementales de Charente-Maritime, Enquête du Commissaire Chardon (1783), 2 J 10, (non publié).
l'État ne pouvait se lancer dans des opérations de quelque nature qu'elles soient sur un littoral où ses agents, sans être oublieux de leur mission de service public, ne méconnaissaient ni l'expérience - et la mémoire - locales, ni la force des intérêts particuliers. En réalité, ils s'attachaient à arbitrer au quotidien entre ces forces, d'autant plus que leurs effectifs limités n'autorisaient pas vraiment la mise en œuvre de la politique littorale par le biais d'une quelconque contrainte. Ajoutons qu'avec le couple Marine-Amirauté, l'Ancien Régime avait à son service une administration unique pour traiter de l'ensemble des problématiques littorales, situation dont on n'a cessé de s'éloigner depuis lors.

\section{LE LITTORAL ENTRE CONVOITISE ET EXPERTISE}

La Révolution française a mis à mal le fragile équilibre qui présidait aux destinées du littoral avant 1789. Dans un premier temps, la priorité résida dans la conduite de la guerre contre les Anglais, dans un contexte de crise économique et de blocus maritime quasi ininterrompu (1793-1815). L’appréhension de ce hiatus administratif est essentielle à la compréhension du contexte de rédaction de la loi de 1807 sur les aménagements littoraux. C'est cette loi dont l'article 33 régit toujours le droit des zones asséchées : les polders. On y lit la consécration de l'initiative et de la responsabilité individuelles, substituées à la puissance publique d'un État alors défaillant. Dans un contexte de blocus et de pénurie de céréales il importait alors de permettre aux capitaux accumulés dans le commerce du sel, du vin, du sucre et des denrées coloniales, d'être mobilisés au service de l'entretien des polders existants et de la colonisation des lais et relais de mer, pour en aménager de nouveaux. L'Empire avait besoin de champs destinés à produire ces grains, dont l'importation était désormais devenue aléatoire.

\section{Exploiter la mer : l'exaltation de l'initiative locale par la monarchie libérale}

Cette évolution majeure du droit, si elle ne semble pas avoir été à l'origine d'un vaste mouvement d'aménagement durant le premier tiers du XIX ${ }^{\mathrm{e}}$ siècle, a bel et bien posé les bases d'une 
appropriation populaire de la question environnementale sur le littoral. La tendance fut accélérée suite au partage des dépouilles de l'Amirauté, par des institutions rivales ou concurrentes: Marine, Domaines, Ponts et Chaussées, Douanes, municipalités, échelons locaux de justice (Sauzeau, à paraître). Les fonds d'archives contemporains, livrent ainsi en miettes, l'histoire des rapports entre les populations riveraines et l'environnement littoral tels qu'on peut les recenser dans les seuls dossiers de l'Amirauté pour le XVIII ${ }^{\mathrm{e}}$ siècle. Le contexte politique des années 1815-1848 a infléchi les rapports entre une autorité divisée et les riverains. En effet, sur les questions frumentaires, la monarchie administrative du XVIII ${ }^{\mathrm{e}}$ siècle était marquée par son paternalisme à l'égard de ses sujets : la sécurité alimentaire était un domaine régalien. La monarchie de Juillet se caractérisait quant à elle par une exaltation de l'initiative individuelle, très marquée par les thèses du libéralisme économique. Le marché était investi de la mission de nourrir les populations et sur le littoral, il était logique du lui confier l'aménagement de nouveaux espaces de production : claires et parcs ostréicoles, pêcheries, polders dédiés à la céréaliculture (Verger, 2005). Dès 1811, à Oléron, un groupe de 4 particuliers obtenait l'autorisation d'endiguer les vasières comprises entre les chenaux d'Arceau et de La Perrotine et en 1833, ils étaient 5 nouveaux demandeurs à assécher les vases à l'avant de cette première «tanne ${ }^{13} »$. Sur le continent, un plan d'endiguement des laisses de mer était soumis à l'autorité préfectorale en 1818, afin de gagner sur la mer de nouveaux champs, entre les chenaux de Brouage et de Mérignac (figure 5 - planche IV). Les correspondances au départ des bureaux des classes de la Marine, tout comme les dossiers instruits par les services des Domaines ou des Ponts et Chaussées témoignent du regain d'intérêt pour l'économie maritime et littorale après 1815. La multiplication des interlocuteurs facilitait les projets des plus entreprenants qui n'hésitaient pas à s'adresser à plusieurs guichets, dans l'espoir d'arracher la décision favorable à un projet d'aménagement ou une quelconque autorisation. Dans un contexte de dérégulation favorable aux détenteurs de capital, cela conduisit à une succession de crises et de conflits. En 1855, sur Oléron, un projet touchant une « par-

13. Archives départementales de Charente-Maritime, Aliénation de terrains domaniaux (1833), 4S 7764, (non publié). celle rive droite du chenal de la Perrotine à endiguer, entraînerait la destruction de claires à huîtres " en foi de quoi on modifie le tracé de l'endiguement ${ }^{14}$. Quinze ans plus tard, l'administration refusera un projet d'endiguement sur la rive gauche du chenal d'Ors, au motif que "l'endiguement des parcelles le long du chenal est une cause de ruine pour ces chenaux et qu'il est de l'intérêt bien entendu de l'État de ne pas aliéner les terrains submersibles pour laisser à la mer montante le plus grand développement possible». Au cours de cette phase du développement littoral, le dialogue entre administrés et autorité consistait à faire jouer le curseur entre les activités maritimes, celles de l'estran et la dynamique des aménagements agricoles (polders). En dépit de la crise des années 1850, le lien entre prise de décision et mémoire détenue par les riverains restait une donnée essentielle du rapport à la côte.

\section{Entre domaine public maritime et construction d'une mémoire officielle}

En 1853, le second Empire vint réaffirmer avec force les principes de domanialité sur le littoral. C'était en quelque sorte un retour en force de l'étatisme d'Ancien Régime. Sous la pression de la croissance démographique, les mouvements de poldérisation continuaient, car il fallait bien nourrir des bouches de plus en plus nombreuses. L'urbanisation progressait aussi, à mesure que les ports développaient leurs infrastructures. Ainsi à La Perrotine, sur l'île d'Oléron, un plan d'alignement daté de 1905 montre une dizaine de parcelles, avec des maisons disposant d'une façade sur le chenal et d'un jardin en contrebas dans la tanne (figure 6). Premier des propriétaires, l'entrepreneur de travaux publics Grossard travaillait pour la Marine, à l'entretien des perrés du chenal. Expression d'une culture du risque maritime, les maisons étaient protégées des submersions côté chenal par une «banquette», aménagée sur un "terre-plein », deux aménagements aujourd'hui disparus et qu'il est question de rebâtir ${ }^{15}$. Comme durant la phase libérale, les dynamiques restaient impulsées par des sociétés littorales, désormais aiguillonnées par un marché à la

14. Archives départementales de Charente-Maritime, Aliénation de terrains domaniaux (1855), 4S 7765, (non publié).

15. Cet aménagement est annexé au scénario produit par le bureau d'études EGIS-Eau, engagé par le conseil général de la Charente-Maritime suite à la tempête Xynthia. 


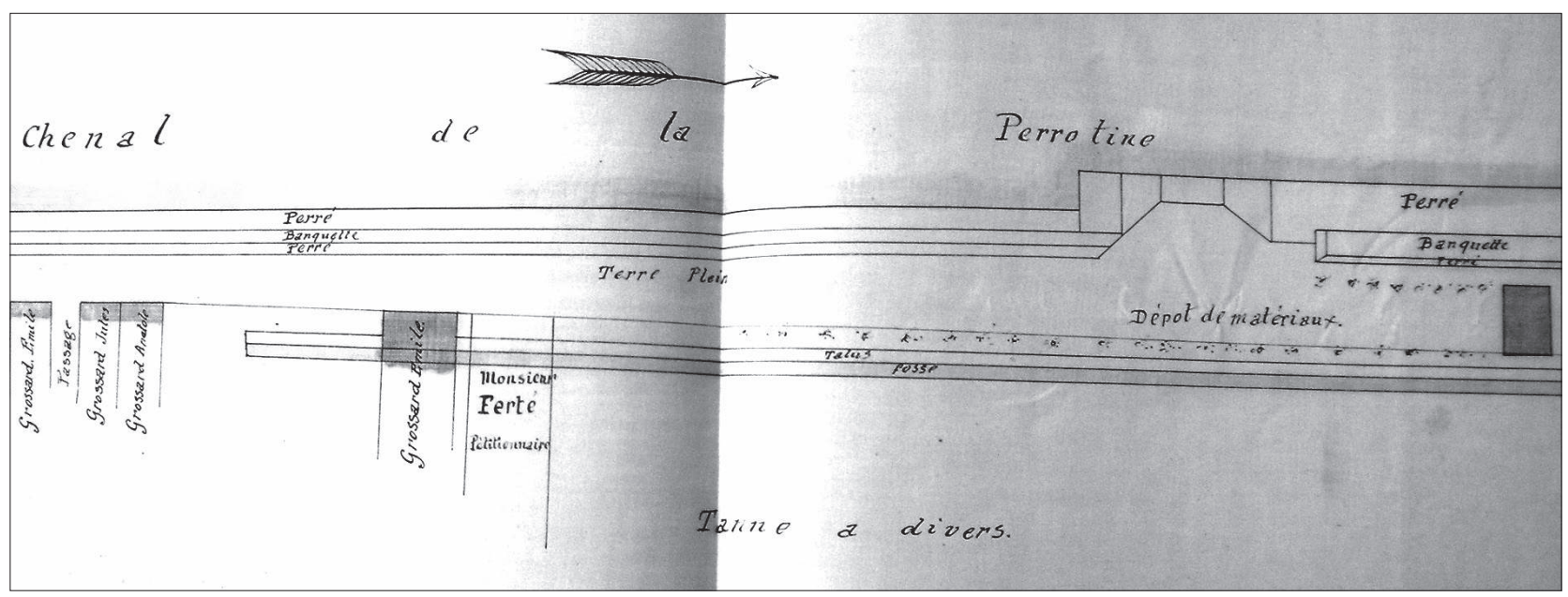

Figure 6 : Plan d'alignement le long du chenal de La Perrotine, île d'Oléron (1905) (source : Archives départementales de la Charente-Maritime, Rapport d'alignement réalisé par l'ingénieur subdivisionnaire, 4S-5138)

Line's map along the La Perrotine channel, Oleron Island (1905)

croissance dopée par la révolution des transports. L'irruption du fait balnéaire à la Belle Époque et sa popularisation entre-deux-guerres vinrent entretenir ce mouvement initié dans les années 1870. Pour la première fois depuis la Révolution française, une phase du développement littoral était associée à d'importants transferts de foncier : les propriétaires et les usages étaient renouvelés. Avec l'entrée dans le $\mathrm{xx}^{\mathrm{e}}$ siècle, l'expérience de la côte et la mémoire du risque s'en trouvèrent peu à peu dissociées. Les sociétés littorales se sont alors engagées sur le chemin qui les a menées d'une sensibilité aux aléas marins à la vulnérabilité qui n'a cessé de s'accentuer depuis. Les nouveaux venus étaient des citadins qui ne s'affrontaient au littoral que durant la belle saison. La place des activités agromaritimes (pêche, ostréiculture, agriculture) toujours importante, était concurrencée par d'autres groupes sociaux. Les destins croisés du petit village des Boucholeurs et de la station balnéaire voisine de Châtelaillon illustrent bien ces mutations différenciées. Implanté sur une plage de galets, produits de l'érosion de la falaise de Châtelaillon, le village est au débouché du canal de Port Punay, exutoire de vastes polders (marais de Voutron) aménagés au XVIII ${ }^{\mathrm{e}}$ siècle. Fondé en $1880^{16}$, l'histoire des Boucholeurs est liée à l'exploitation de l'huître portugaise. Le site quoique reconnu « presque toujours envahi par la mer lors

16. Archives départementales de Charente-Maritime, Rapport d'ingénieur suite à la demande des habitants de Port Punay d'établissement d'un ouvrage d'abri (1907), 4S-3863, (non publié). des mauvais temps », comptait "une soixantaine de feux et 300 habitants $^{17}$ " en 1950, sous la protection rudimentaire d'une palissade de traverses de chemin de fer (figure 7). Quoique de fondation récente, ce village détenait - et conserve - une vraie culture du risque maritime. En revanche dès 1900 à Châtelaillon, on s'alarmait de l'érosion : « $\mathrm{Ce}$ qui reste de dunes est recouvert par les nombreuses constructions de ceux qui forment la station balnéaire de Châtelaillon, une digue devient nécessaire pour rassurer la population et pour éviter la destruction de cette petite ville ${ }^{18}$. " Dans de tels lieux, investis par des populations sans racines maritimes, les ingénieurs et conducteurs des Ponts et Chaussées ont été les ultimes dépositaires de la mémoire du risque. Que ce soit face aux changements graduels ou aux événements extrêmes, l'histoire du lieu - et de ses aménagements - s'imposait encore comme préalable aux dossiers instruits par ces spécialistes. Au terme d'un siècle de développement littoral, c'est sur ces mêmes bases que se déroulèrent les grands travaux à l'époque de la Reconstruction, dernières interventions majeures sur les digues et les dispositifs hydrauliques.

17. Archives départementales de Charente-Maritime, Perré des Boucholeurs : conférence entre le service hydraulique et le génie rural (1950), 4S-3785 (non publié).

18. Archives départementales de la Charente-Maritime, Projet de constitution d'une association syndicale autorisée pour la défense de la côte de Châtelaillon (1900), 4S-4418, (non publié). 


\section{UN LITTORAL VULNÉRABLE, SANS RACINES NI MÉMOIRE}

À l'égard de la mémoire du risque et de l'expérience de la mer, la période qui s'ouvre avec les années 1960 reste marquée par une accumulation d'illusions. La faible fréquence des événements extrêmes est un facteur déterminant (Garnier, 2010). Elle inspire un fort sentiment de confiance à une société désormais tout entière acquise au désir de rivage. La mémoire des événements extrêmes est renvoyée à sa désuétude, les habitants enracinés, qui en sont dépositaires, sont submergés par un progrès dont ils tirent parfois profit, en vendant un bout de champ, mais pas systématiquement. L'essor du tourisme balnéaire consacre l'appropriation des espaces avec vue sur la mer, au profit d'une seule activité : ports de plaisance, boutiques et restaurants saisonniers, lotissements, villages de vacances, camping sous les pins, le tout à deux pas des plages desservies par une voirie et des parkings tracés dans les dunes (Vye, 2010). C'est une nouvelle société qui se construit en quelques décennies sur le littoral. La croissance est forte dans ces zones, à la fois attractives pour les activités tertiaires et convoitées par le flux des résidents secondaires retraités, de plus en plus nombreux dans nos sociétés vieillissantes. Si les activités primaires ne disparaissent pas, elles sont néanmoins marquées par des phénomènes de concentration qui réduit le nombre d'actifs - et de foyers - porteurs d'une réelle culture maritime. Une population neuve s'approprie ces territoires, au moment même où d'autres mutations sont à l'œuvre. Au début des années 1970, l'univers des ingénieurs est transformé par le numérique, qui porte alors un rude coup à la place de l'expérience - et de l'histoire, celle des événements autant que celle des aménagements - dans les grandes écoles qui les forment. Désormais, on accorde plus de crédit à la modélisation numérique d'un avenir probable, qu'à la connaissance - et au retour d'expérience - d'un passé certain. À compter des années 1980, l'urbanisation du littoral va s'effectuer dans cet univers scientifique et technologique indépassable. Dans le cadre des lois de décentralisation, cette génération d'E-ingénieurs a cogéré l'urbanisation, en face à face avec les élus locaux et leurs services techniques. Sous le contrôle des services de l'État, ils ont multiplié les aménagements et les documents d'urbanisme. Sans considération

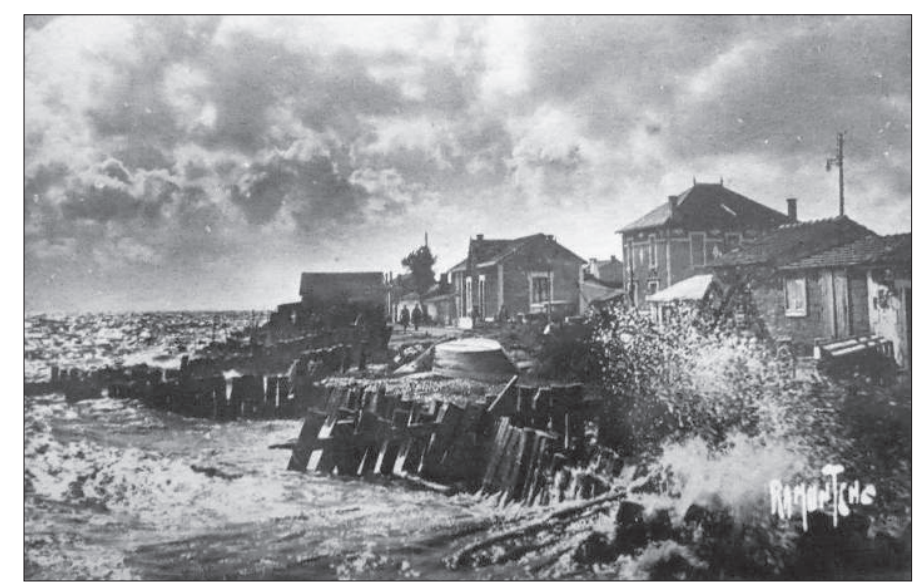

Figure 7 : Carte postale du village des Boucholeurs vers 1930 (source : collection privée)

Postcard of Boucholeurs village around 1930

pour le caractère agricole des polders, on a alors bâti en zone submersible, dans le respect de chartes paysagères qui prohibaient les étages (Vinet et al., 2011) et alors même que les plans de prévention des risques alertaient sur l'aléa de submersion sans la moindre référence aux événements, oubliés...

Xynthia est finalement venue frapper une société inconsciente de sa vulnérabilité, enivrée par le marché et rassurée par sa foi dans un progrès et une sécurité partagée par les simples citoyens, les élus et les élites. Dans un tel contexte, il y avait peu de chance pour que la catastrophe naturelle ne se double pas d'une catastrophe humaine. Quant à la gestion post-catastrophe, elle se serait honorée d'une enquête «à la Colbert » dans les zones submergées par Xynthia, pour collecter la parole des sinistrés, des riverains et des acteurs du littoral, avant de prendre une décision éclairée. La sociologie des désastres enseigne que la réflexion et l'enquête sur le sens (signification et valeur) de la catastrophe permet de gérer les réactions de résistance. Or les autorités considèrent systématiquement ce travail comme « hors sujet» (Signorelli, 1992). En dépit de la révolution balnéaire, la permanence de racines paysannes dans les villages côtiers est pourtant réelle : elle offrait un levier que les autorités n’ont pas saisi. En dépit de la rareté de leurs données instrumentales sur la submersion, les autorités ont défendu leur monopole strict sur le retour d'expérience. Paradoxalement, les habitants qui avaient effectivement expérimenté l'événement n’ont eu d'autres interlocuteurs que des cellules psycholo- 
giques. C'est pourquoi lorsque dans l'urgence, nous avons expérimenté un protocole destiné à recueillir pour la structurer, la parole des habitants enracinés, cela a été bien accueilli. La méthode a été expérimentée sur un espace laboratoire, les rives du chenal de la Perrotine (île d'Oléron) au lendemain de l'annonce des zonages en avril 2010. Le but était de documenter la question des rapports entretenus entre histoire, mémoire et gestion des défenses contre la submersion marine. Nous avons mené trois enquêtes, avant d'en confronter les résultats. La mobilisation des cartes anciennes de la collection " Paysages et patrimoine du CentreOuest atlantique ${ }^{19}$ », a permis de constituer un atlas dynamique du paysage. Nous avons déterminé les grandes étapes de la construction du territoire, en estimant la date des premiers noyaux de peuplement permanent, en précisant les aménagements auxquels ils avaient donné lieu et en reconstituant leurs dynamiques respectives. Ce travail de géohistorien s'est ensuite confronté à la parole des habitants présents dans la nuit du 28 février 2010. Où, comment et à quelle heure les aménagements de défense contre la submersion avaient-ils failli ? Chaque habitant présent la nuit du sinistre a pu témoigner de son expérience vécue de Xynthia : chronologie des faits, observation de la submersion, résultats et impact. Tout a été consigné sur des procès-verbaux, datés et signés. Très vite, et de manière inattendue, les habitants enracinés ont proposé leur vision des facteurs aggravants de la submersion. Le dernier demi-siècle a été décortiqué, documents à l'appui (photos et cartes postales anciennes, plans et archives privées). Trois ensembles documentaires ont ainsi pu être constitués : une description dynamique de la submersion du 28 février 2010; un recensement mémoriel des facteurs aggravants de la catastrophe; une biographie du territoire basée sur les cartes anciennes. La grande cohérence des trois corpus a finalement permis de dresser un diagnostic argumenté du passage historique de la conscience à l'inconscience, de la connaissance du risque à la vulnérabilité contemporaine (Sauzeau, 2010). Loin de se dresser en procureur à la recherche de responsables, nous sommes parvenus à poser un diagnostic. La mesure de la vulnérabilité en termes d'imprudences - constructions hasardeuses - et de

19. Base de données en ligne : [http://www.mshs.univ-poitiers.fr/paysagespatrimoine]. négligences - absence d'entretien - a ainsi permis de suggérer des pistes de réversibilité. Au-delà, la pertinence de tels résultats à convaincu la commune de Saint-Georges-d'Oléron d'annexer à son Plan Communal de Sauvegarde un tableau des événements de référence (tableau 1) identifiés sur les différents points du territoire communal. Cette initiative fournit un exemple concret pour associer étroitement les événements révélateurs du risque à la traditionnelle cartographie des aléas qui figure depuis des années dans les Plans de Prévention des Risques Littoraux.

\section{Conclusion}

Sur le littoral, la question de la mémoire du risque et de sa place dans le processus de décision est utilement éclairée par l'examen des rapports entretenus par l'autorité et les sociétés riveraines. À cet égard, depuis l'avènement de l'État moderne, on distingue nettement quatre phases. Avant 1789 puis à nouveau, du Second Empire au début de la Ve République (1850-1960), l'État centralisé s'est appliqué à gérer son littoral. Avec une administration maritime unique au maillage très lâche, la monarchie absolue n'avait d'autre choix que de valoriser l'expérience du rivage, la mémoire de ses habitants. Après 1850, les administrations beaucoup plus développées dont disposaient les régimes successifs ont intégré cette mémoire et cette histoire à la formation et à la pratique de leurs agents. L'État incluait réellement le littoral dans son périmètre régalien - nouveau passage d'une souveraineté nominale à une souveraineté effective - et son souci d'efficacité passait par la maîtrise de toutes les sources d'information avant de décider et d'agir. Cet apprentissage de la mer est aussi passé par des phases moins volontaristes, marquées par le recul de l'État. La loi de 1807 a ainsi posé les bases d'un demi-siècle de politique libérale dont la monarchie de Juillet fut le point d'orgue. Polders, aménagements portuaires, parcs et claires ostréicoles, pêcheries, le littoral devint alors un petit far-west, une zone d'appropriation incertaine dans la mesure où les prétentions domaniales de l'État étaient subordonnées au développement économique. L'analogie avec la période 1960-2010 est-elle pertinente? Les moteurs économiques diffèrent (la production agromaritime au $\mathrm{XIX}^{\mathrm{e}}$ siècle; le tourisme balnéaire 


\begin{tabular}{|c|c|c|c|c|c|c|c|}
\hline & \multicolumn{5}{|c|}{ côte à marées (Est), influencées par les houles } & \multicolumn{2}{|c|}{ côtes à houle (Ouest) } \\
\hline $\begin{array}{r}\text { Lieux } \\
\text { Date, année }\end{array}$ & Boyardville & le Douhet & Plaisance & Foulerot & L'Ileau & Chaucre & Ponthezière \\
\hline \multirow{3}{*}{$\begin{array}{l}24-26 \text { fév. } \\
1838\end{array}$} & cote $4 \mathrm{~m} 75$ & & & & salines & & \\
\hline & & dunes & dunes & dunes & taillées canal & dunes, canal & dunes, canal \\
\hline & & & & & salines & champs & champs \\
\hline \multirow{3}{*}{$\begin{array}{l}\text { maline de janv- } \\
\text { fév. } 1846\end{array}$} & & & La Durandière & & & & \\
\hline & & dunes & La Durandière & & & & \\
\hline & & & & & & & \\
\hline \multirow{3}{*}{$\begin{array}{l}\text { Hiver 1854- } \\
\quad 1855\end{array}$} & & & & & & & \\
\hline & & & & & & dunes, canal & dunes, canal \\
\hline & & & & & & champs & champs \\
\hline \multirow{3}{*}{$\begin{array}{l}\text { Hiver } 1860- \\
\quad 1861\end{array}$} & & & & & & & \\
\hline & & & & & & dunes, canal & dunes, canal \\
\hline & & & & & & champs & champs \\
\hline \multirow{3}{*}{$\begin{array}{c}\text { Hiver 1872- } \\
1873\end{array}$} & & & & & & & \\
\hline & & & & & & dunes, canal & dunes, canal \\
\hline & & & & & & \multicolumn{2}{|c|}{ Champs sous $1 \mathrm{~m}$ d'eau env. } \\
\hline \multirow{3}{*}{$\begin{array}{c}26-27 \\
\text { oct. } 1883\end{array}$} & cote $4 \mathrm{~m} 20$ & & & & & & \\
\hline & & & & & & & \\
\hline & & & & & & & \\
\hline \multirow[b]{2}{*}{27 mars 1888} & & & & & & & \\
\hline & jetée, quais & & & & & & \\
\hline 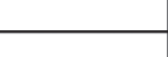 & & & & & & & \\
\hline \multirow{2}{*}{$\begin{array}{l}22-23 \text { fév. } \\
1935\end{array}$} & nbx.arbres & \multicolumn{2}{|c|}{ digue de défense arrachée } & & & & \\
\hline & & & & & & & \\
\hline \multirow{3}{*}{$\begin{array}{c}\text { 13-14 fév. } \\
1937\end{array}$} & & \multicolumn{3}{|c|}{ route le Douhet Plaisance $(1 \mathrm{~m}$ d'eau $)+$ qq.ha. de bois } & & & \\
\hline & & & \multicolumn{2}{|c|}{ plage et dune ravagée sur $500 \mathrm{~m}$} & & & \\
\hline & & & & & & & \\
\hline \multirow{3}{*}{$\begin{array}{l}\text { 14-15 fév. } \\
1957\end{array}$} & & & & & & & \\
\hline & & \multicolumn{4}{|c|}{ les eaux se sont déversées dans le marais en contrebas ( 600 ha.subm.) } & & \\
\hline & & \multicolumn{3}{|c|}{ route le Douhet Plaisance emportée sur $1 \mathrm{~km}$} & & & \\
\hline & & & & & & & \\
\hline \multirow[t]{2}{*}{ 28-mars-63 } & & & & & & & \\
\hline & & \multicolumn{3}{|c|}{ route le Douhet Plaisance à nouveau menacée } & & & \\
\hline \multirow{3}{*}{$\begin{array}{l}27-28 \text { fév. } \\
2010\end{array}$} & cote $4 \mathrm{~m} 30$ & & & & & & \\
\hline & & & & & & & \\
\hline & & & & & & & \\
\hline
\end{tabular}

Tableau 1 : Inventaire historique des événements extrêmes à Saint-Georges-d'Oléron Historical inventory of natural events at Saint-Georges-d'Oléron submersion destruction inondation au $\mathrm{xx}^{\mathrm{e}}$ siècle) mais au chapitre du désengagement étatique, on retrouve l'adhésion à un libéralisme économique qui atteint ses limites face à la mer et à ses dangers. Quel retour sur expérience l'historien peut-il suggérer? Le Second Empire, avec le décret sur les pêches côtières (1853), a restauré le domaine public maritime. Ce fut - entre autre - un levier de soutien à l'expérience ostréicole, la solution pour dépasser le problème d'une ressource que la surpêche avait pillé. Cette issue favorable à une crise majeure prouve qu'il n'est pas impossible de concilier développement économique et réglementation, dans un dialogue permanent avec les acteurs de terrain. La sécurité des villes et des villages du littoral français peut-elle bénéficier des acquis de l'expérience des derniers habitants enracinés et de nos archives? Principal outil à la disposition de territoires invités à repenser leur politique de prévention 
du risque, l'actuel guide d'élaboration des Plans de préventions des risques littoraux - paru en 1997 fait l'impasse sur ces questions. La nouvelle version, en préparation au Centre d'Études Techniques Maritimes et Fluviales devrait combler cette lacune. Il s'agit de les mobiliser pour restaurer la conscience du risque qui existait avant la grande amnésie du second $\mathrm{xx}^{\mathrm{e}}$ siècle. Une politique de mémoire renouvellerait les bases de la cohabitation avec la mer et ses colères. La vigilance devrait inspirer des réglementations, des aménagements, une pédagogie, une politique d'information, de prévention, d'alerte et de réaction face au danger. La «vue sur la mer » expose son bénéficiaire au risque de submersion. Il faut le dire, le montrer et en accepter l'impact sur le marché immobilier. Xynthia offre l'opportunité d'inventer une politique citoyenne différente de la voie empruntée en 2010, qui n'interrompt nullement le travail de sape de notre vigilance collective. Une fois les zones sinistrées vidées de leurs habitants, la mer n'en restera pas moins dangereuse et libre de frapper ailleurs, dans les mêmes conditions.

\section{Bibliographie}

ACERRA M., 1993. Rochefort et la construction navale française, 1661-1815, Paris, Librairie de l'Inde, première partie, 930 p.

Corbin A., 1988. Le territoire du vide. L'occident ou le désir de rivage, Paris, Flammarion, $400 \mathrm{p}$.

Garnier E., 2010. 500 ans de vimers sur le littoral de Poitou-Charentes : risque et vulnérabilité des sociétés, dans Sauzeau T. (dir.), Comprendre Xynthia, expliquer un phénomène, Journée d'études de la Région PoitouCharentes, Poitiers, p. 11-17 [http://fr.calameo.com/ books/00050825208efca445157].

PÉRET J., 2006. Naufrages et pilleurs d'épaves sur les côtes charentaises, La Crèche, Geste éditions, 263 p.

Sauzeau T., 2010. La Perrotine-Boyardville, rapport d'expertise suite à la tempête Xynthia, Le journal des propriétaires de l'île d'Oléron, n 104, p. 10-16.

SAUZEAU T., à paraître. De l'Amirauté à l'Inscription Maritime, «l'estran compliqué » de la Saintonge maritime (17601865), nº spécial, L’Amirauté, Revue d'Histoire Maritime, Paris, PUPS.

Schnackenbourg C., 1975. L'Amirauté de France à l'époque de la monarchie administrative (1662-1792), Thèse de droit, Paris II, 2 tomes, $651 \mathrm{p}$.

Signorelli A., 1992. Catastrophes naturelles et réponses culturelles, Terrain, $\mathrm{n}^{\circ} 19$ [http://terrain.revues.org/index3052. $h t m l]$.

Taillemite E., 2002. Dictionnaire des marins français, Paris, Tallandier, $524 \mathrm{p}$.

Verger F., 2005. Marais et estuaires du littoral français, Paris, Belin, 335 p.

Vinet F., Defossez S., Leclere J.-R., 2011. Comment se construit une catastrophe, dans Mercier D., Acerra M. (dir.), Xynthia, une tragédie prévisible, Nantes, Place Publique, Hors-Série, p. 9-16.

Vye D., 2010. Quand Xynthia fait rimer attractivité du littoral avec vulnérabilité, dans SAuzeau T. (dir.), Comprendre Xynthia, expliquer un phénomène, Journée d'études de la Région Poitou-Charentes, Poitiers, p. 18-21 [http://fr.calameo.com/ books/00050825208efca445157].

Zysberg A., 1995. La soumission du rivage aux volontés de l'État royal, dans Acerra M., Poussou J.-P., Vergé-FranCeschi M., Zysberg A. (dir.), État, marine et société, hommages à Jean Meyer, Paris, PUPS, p. 439-455. 